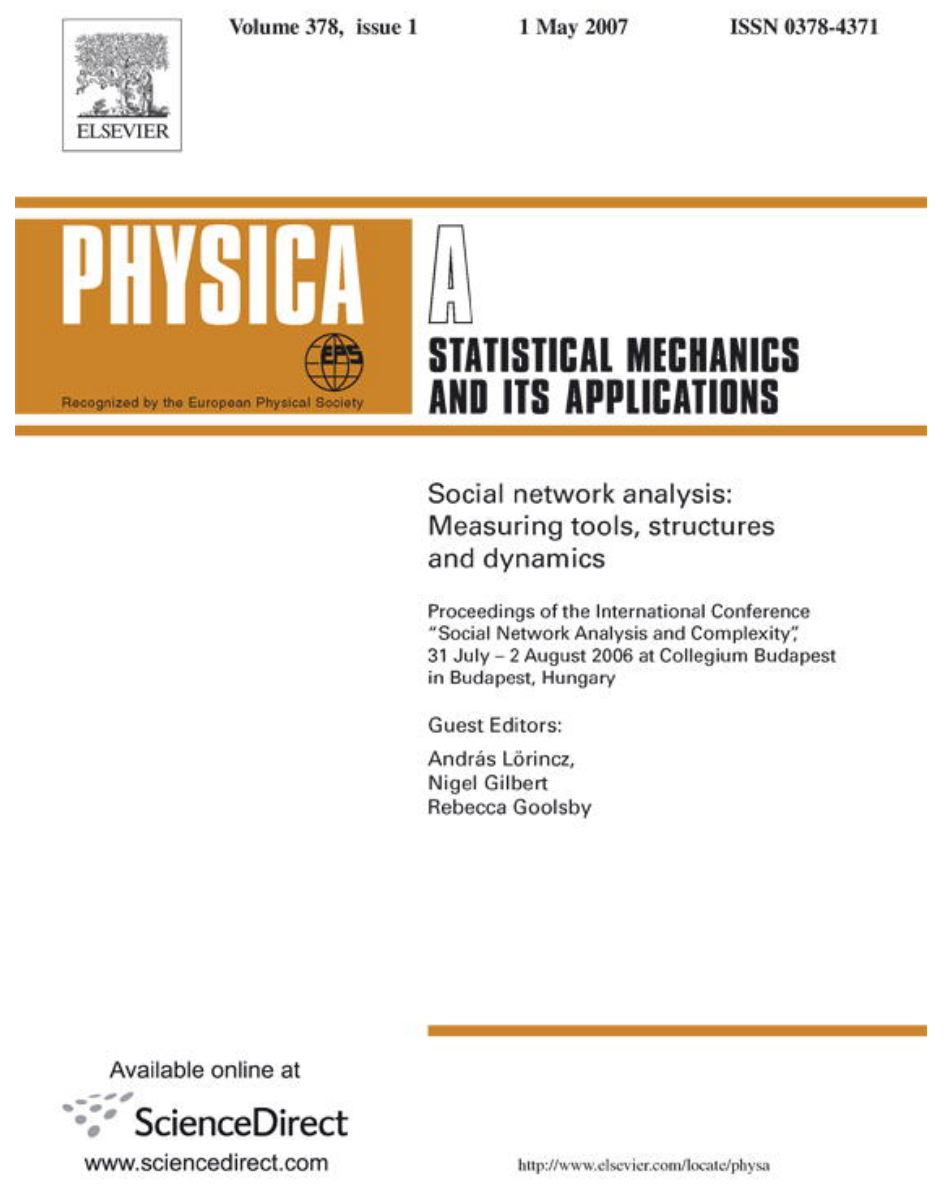

This article was originally published in a journal published by Elsevier, and the attached copy is provided by Elsevier for the author's benefit and for the benefit of the author's institution, for non-commercial research and educational use including without limitation use in instruction at your institution, sending it to specific colleagues that you know, and providing a copy to your institution's administrator.

All other uses, reproduction and distribution, including without limitation commercial reprints, selling or licensing copies or access,

or posting on open internet sites, your personal or institution's website or repository, are prohibited. For exceptions, permission may be sought for such use through Elsevier's permissions site at: 


\title{
Percolation theory and fragmentation measures in social networks
}

\author{
Yiping Chen ${ }^{\mathrm{a}}$, Gerald Paul ${ }^{\mathrm{a}}$, Reuven Cohen ${ }^{\mathrm{b}}$, Shlomo Havlin ${ }^{\mathrm{c}, *}$, \\ Stephen P. Borgatti ${ }^{\mathrm{d}}$, Fredrik Liljeros ${ }^{\mathrm{e}}$, H. Eugene Stanley ${ }^{\mathrm{a}}$ \\ ${ }^{\mathrm{a} C}$ enter for Polymer Studies, Boston U niversity, Boston, M A 02215, USA \\ ${ }^{b} D$ epartment of Electrical and Computer Engineering, Boston U niversity, B oston, M A 02215, USA \\ ${ }^{\mathrm{C}} \mathrm{M}$ inerva $\mathrm{C}$ enter and Department of Physics, Bar-I lan U niversity, 52900 R amat-Gan, I srael \\ ${ }^{\mathrm{d} D}$ epartment of Org. Studies, B oston College, Chestnut H ill, M A 02467, U SA \\ 'D epartment of Sociology, Stockholm U niversity, S-106 91 Stockholm, Sweden
}

Available online 18 December 2006

\begin{abstract}
We study the statistical properties of a recently proposed social networks measure of fragmentation $F$ after removal of a fraction $q$ of nodes or links from the network. The measure $F$ is defined as the ratio of the number of pairs of nodes that are not connected in the fragmented network to the total number of pairs in the original fully connected network. We compare this measure with the one traditionally used in percolation theory, $\mathrm{P}_{\infty}$, the fraction of nodes in the largest cluster relative to the total number of nodes. Using both analytical and numerical methods, we study Erdős-Rényi (ER) and scale-free (SF) networks under various node removal strategies. We find that for a network obtained after removal of a fraction $q$ of nodes above criticality, $\mathrm{P}_{\infty} \approx(1-\mathrm{F})^{1 / 2}$. For fixed $\mathrm{P}_{\infty}$ and close to criticality, we show that $1-\mathrm{F}$ better reflects the actual fragmentation. For a given $P_{\infty}, 1-F$ has a broad distribution and thus one can improve significantly the fragmentation of the network. We also study and compare the fragmentation measure $F$ and the percolation measure $\mathrm{P}_{\infty}$ for a real national social network of workplaces linked by the households of the employees and find similar results.
\end{abstract}

(C) 2006 Elsevier B.V. All rights reserved.

K eywords: Social network; Fragmentation; Percolation theory

\section{Introduction}

Complex networks can be used to model many physical, sociological and biological systems and have attracted much attention in recent years [1-14]. Among the problems related to complex networks, the fragmentation of networks has been extensively studied [5-11]. The problem is defined as finding the statistical properties of the fragmented networks after removing nodes (or links) from the original fully connected network using a certain strategy. Many different removal strategies have been developed for various purposes, e.g., mimicking the real world network failures, improving the effectiveness of network disintegration, etc.

*Corresponding author. Tel.: +9723531 8436; fax: + 97235357678.

E-mail address: havlin@ophir.ph.biu.ac.il (S. Havlin). 
Examples include random removal (RR) strategy, the high degree removal (HDR) strategy and the high centrality removal strategy $[8,15-17]$.

Recently, a new measure of fragmentation has been developed in social network studies [18]. Suppose a fully connected network of $\mathrm{N}$ nodes is fragmented into separate clusters [19] by removing $\mathrm{m}$ nodes following a certain strategy. We define $q \equiv m / N$ the ratio of nodes removed and $p \equiv 1-q$ the ratio of existing nodes. The degree of fragmentation $\mathrm{F}$ of the network is defined as the ratio between the number of pairs of nodes that are not connected in the fragmented network to the possible number of pairs in the original fully connected network. Suppose there are $\mathrm{m}$ clusters in the fragmented network, since all members of a cluster are, by definition, mutually reachable, the measure $F$ can be written as follows [18]:

$$
\mathrm{F} \equiv 1-\frac{\sum_{\mathrm{j}=1}^{m} N_{j}\left(\mathrm{~N}_{\mathrm{j}}-1\right)}{\mathrm{N}(\mathrm{N}-1)} \equiv 1-\mathrm{C} .
$$

Here, $\mathrm{N}_{\mathrm{j}}$ is the number of nodes in cluster $\mathrm{j}, \mathrm{m}$ is number of clusters in the fragmented network, and $\mathrm{N}$ the number of nodes in the original fully connected network. For an undamaged network, $F=0$. For a totally fragmented network, $F=1$. The quantity $C$ defined in Eq. (1) can be regarded as the "connectivity" of the network. When $C=1$ the network is fully connected while for $C=0$ it is fully fragmented.

In this paper, we study the statistical behavior of $F \equiv 1-C$ using both analytical and numerical methods and relate it to the traditional measure, the relative size of the largest cluster $\mathrm{P}_{\infty}$, used in percolation theory. In this way, we are able to obtain analytical results for the fragmentation $F$ of networks. We study two removal strategies: the random removal $(R R)$ strategy which removes randomly selected nodes and the high degree removal (HDR) strategy which targets and removes nodes with highest degree. The HDR strategy first removes the node with the highest degree, and then the second highest and so on. These two strategies are commonly used in models representing random and targeted attacks in real world networks $[2,5-7]$.

\section{Theory}

Traditionally, in analogy to percolation, physicists describe the connectivity of a fragmented network by the ratio $\mathrm{P}_{\infty} \equiv \mathrm{N}_{\infty} / \mathrm{N}$ (called the incipient order parameter) between the largest cluster size $\mathrm{N}_{\infty}$ (called the infinite cluster) and $N$. Many properties have been derived for this measure [5,20,21]. For example, in random networks, $P_{\infty}$ undergoes a second order phase transition at a threshold $p_{c}$. Below $p_{c}, P_{\infty}$ is zero for $N \rightarrow \infty$, while for $p>p_{c}, P_{\infty}$ is finite. This occurs for both RR and HDR in random networks [5-7,20]. The threshold parameter $p_{c}$ depends on the degree distribution, the network topology, and the removal strategy [5-7,20,21]. The specific way that $\mathrm{P}_{\infty}$ approaches zero at $p_{c}$ depends on the network topology and removal strategy but not on details such as $p_{c}$. In scale free networks, where the degree distribution $p(k) \sim k^{-\lambda}$ and $2<\lambda<3$, it has been found that $p_{c} \rightarrow 0$ for RR strategy [5] while $p_{c}$ is very high for HDR strategy [6,7]. For $\lambda>3$ and RR, $p_{c}$ is finite.

Next, we show simulation results of removing nodes in both strategies (RR and HDR) on ER and scale free networks. Fig. 1 shows the behavior of $C(\equiv 1-F)$ and $P_{\infty}$ versus $q$ for Erdős-Rényi (ER) and scale-free (SF) networks with RR (Fig. 1(a) and (b)) and HDR (Fig. 1(c) and (d)) strategies. As seen in Fig. 1(a), the network becomes more fragmented when $q$ increases and both measures drop sharply at $q_{c}=1-p_{c}$. Note that $C$ shows a transition similar to $P_{\infty}$ at $p=p_{c}$; however, above $q_{c}, C$ becomes more flat in contrast to $P_{\infty}$, indicating the effect of connectivity in the small clusters which do not effect $\mathrm{P}_{\infty}$.

In contrast to Fig. 1(a), the transition in Fig. 1(b) is not so sharp and therefore $C$ and $\mathrm{P}_{\infty}$ do not show a collapse together. The reason is that for $\lambda=2.5$ there is no transition at $\mathrm{q}<1$ [6] and for $\lambda=3.5, \mathrm{P}_{\infty}$ falls much less sharply compared to ER [22]. For HDR shown in Fig.1(c) and (d), the transition is again sharp since after removing high degree nodes the network becomes similar to ER networks, which do not have high degree nodes [7].

When $p>p_{c}$ and not too close to $p_{c}$, following percolation theory, the infinite cluster dominates the system and $\mathrm{P}_{\infty} \approx \mathrm{p}$, i.e., most of unremoved nodes are connected. Thus, we assume that the small clusters will have a small effect on $\mathrm{C}$ compared to the largest one. Using this assumption, Eq. (1) can be 
a

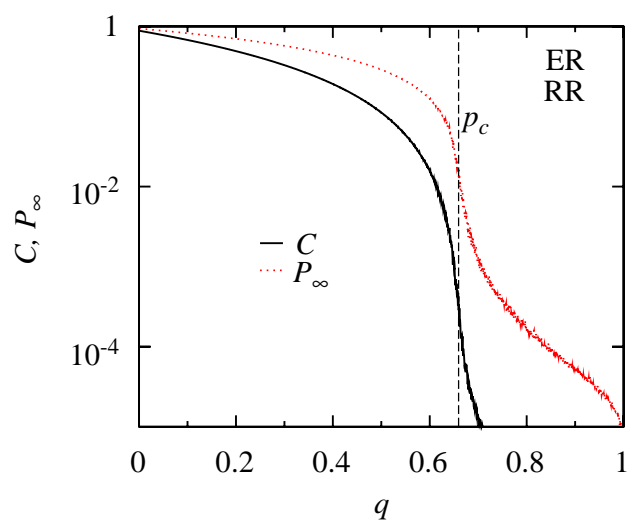

C

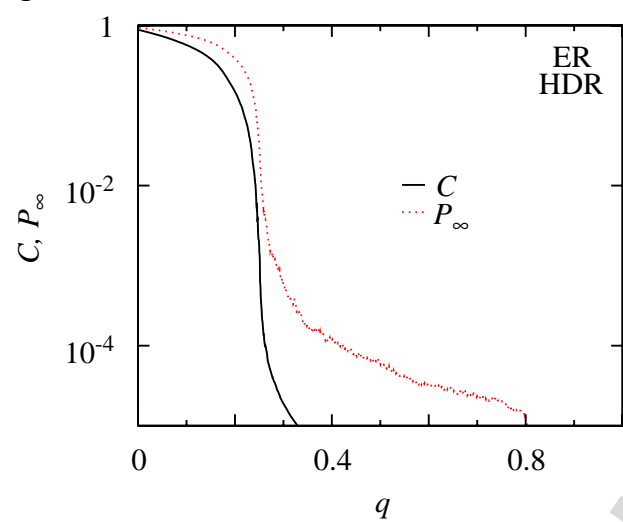

b

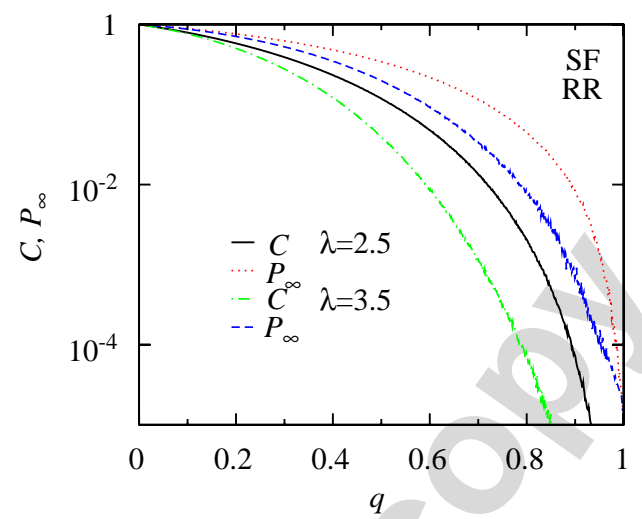

d

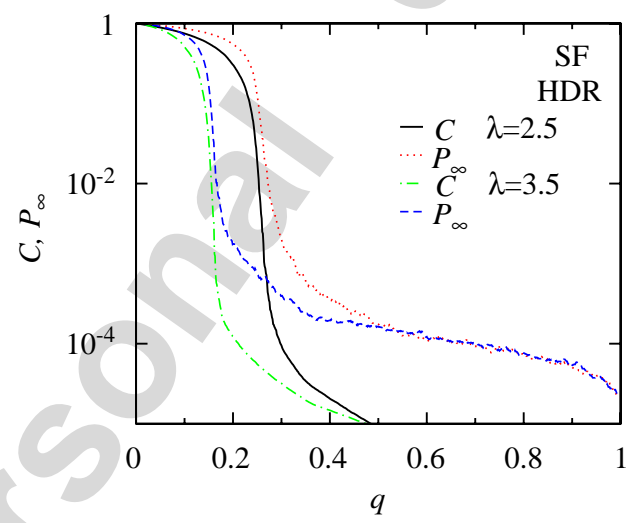

Fig. 1. The behavior of $C$ and $P_{\infty}$ versus $q$ on ER and SF networks. For ER networks, $N=200000$ and $\langle k\rangle=3$. For SF networks, $\mathrm{N}=80000$. The graphs are (a) RR strategy on ER networks, (b) RR strategy on SF networks, (c) HDR strategy on ER networks and (d) HDR strategy on SF networks.

written as

$$
\mathrm{C} \equiv 1-\mathrm{F} \equiv \frac{\sum_{\mathrm{j}=1}^{\mathrm{i}} \mathrm{N}_{\mathrm{j}}\left(\mathrm{N}_{\mathrm{j}}-1\right)}{\mathrm{N}(\mathrm{N}-1)} \approx \frac{\mathrm{N}_{\infty}\left(\mathrm{N}_{\infty}-1\right)}{\mathrm{N}(\mathrm{N}-1)} \approx \frac{\mathrm{N}_{\infty}^{2}}{\mathrm{~N}^{2}} \approx \mathrm{P}_{\infty}^{2}
$$

Therefore, we expect $\mathrm{P}_{\infty}$ and $\mathrm{C}$ have the relationship $\mathrm{P}_{\infty} \approx \mathrm{C}^{1 / 2}$ when $\mathrm{p}>\mathrm{p}_{\mathrm{c}}$ (but not too close to $\mathrm{p}_{c}$ ). When $p \leqslant p_{c}$, the infinite cluster loses its dominance in the system and $P_{\infty} \sim \ln (N) / N \rightarrow 0$ for large $N$ [7]. Here significant variations between $P_{\infty}$ and $C^{1 / 2}$ are expected, as indeed seen in Fig. 2.

\section{Simulations}

We test by simulations the relationship $C \sim P_{\infty}^{2}$ derived for $p>p_{c}$ in Eq. (2). In Fig. 2(a) we plot $P_{\infty}$ versus $C^{1 / 2}$ for RR strategy in ER networks and for several values of $p$. As predicted by Eq. (2), the plot of $P_{\infty}$ versus $C^{1 / 2}$ yields a linear relationship with slope equal to 1 when $p>p_{c}=1 /\langle k\rangle=\frac{1}{3}$. The range of $P_{\infty}$ and $C^{1 / 2}$ for $p=0.4$ is due to the variation of $P_{\infty}$ for a given $p$ and the same variation appears for $C^{1 / 2}$ showing that the infinite cluster dominates and Eq. (2) is valid. However, when $p$ drops close to $p_{c}=\frac{1}{3}$, the system approaches criticality and the one-to-one correspondence between $C^{1 / 2}$ and $P_{\infty}$ is not so strong. This variation is attributed to the presence of clusters other than the infinite one, which influence $C$ but not $P_{\infty}$.

Similar behavior is observed for RR strategy in SF networks with $\lambda=3.5$ shown in Fig. 2(b). For $\lambda=3.5$, the variation in $C^{1 / 2}$ emerge close to $p_{c}=0.2$. However, for $\lambda=2.5$, percolation theory suggests that $p_{c}$ approaches 0 for large systems. As a result, no significant variation is observed even when $\mathrm{P}_{\infty}$ is as small as 
a

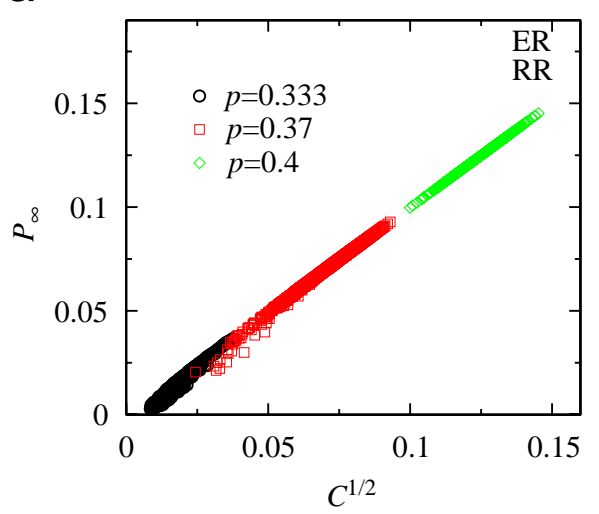

C

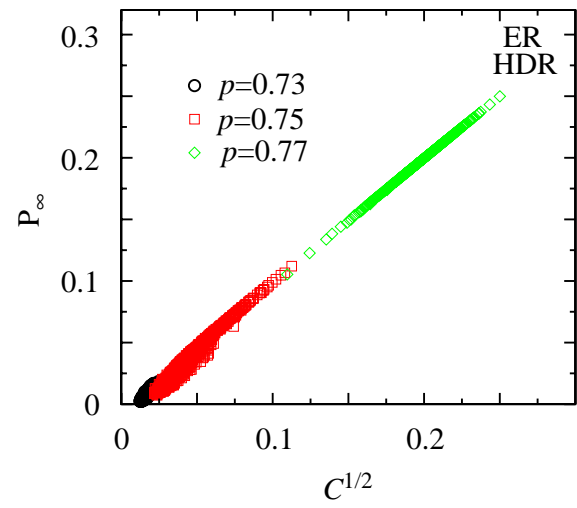

b

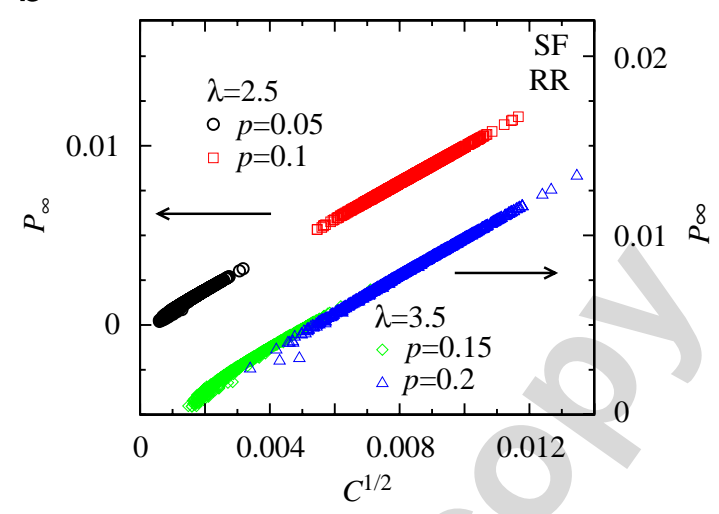

d

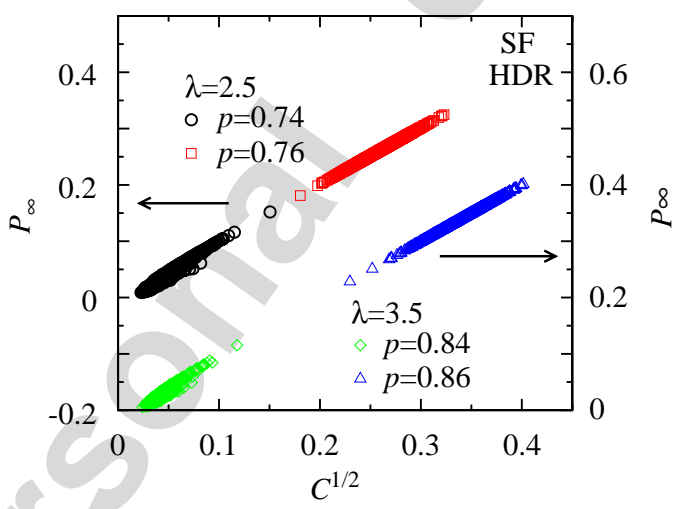

Fig. 2. Relationship between $C^{1 / 2}$ and $P_{\infty}$ for ER and $S F$ networks with system size $N=50000$. For ER networks, the average degree $\langle\mathrm{k}\rangle=3$, and for SF networks, $\lambda=2.5$ and 3.5. The graphs are (a) RR strategy on ER networks, (b) RR strategy on SF networks, (c) HDR strategy on ER networks and (d) HDR strategy on SF networks.

$5 \times 10^{-4}$. This observation supports that the SF networks with $\lambda<3$ are quite robust in sustaining its infinite cluster against random removal [5]. Fig. 2(c) and (d) shows the results for HDR strategy in ER and SF networks. For this targeted strategy, the variation of $C^{1 / 2}$ and $\mathrm{P}_{\infty}$ shows up at significantly higher $\mathrm{p}$ compared to the random case, indicating that the infinite cluster breaks down easier under HDR attacks for both ER and SF networks, as seen also in Fig. 1 . At this point, the SF network with $\lambda=2.5$ becomes no longer as robust as in the random case, as it can be clearly observed in the large variation at $\mathrm{P}_{\infty} \approx 0.05$.

To further investigate the characteristics of the variation of $C$ for a given $P_{\infty}$, we calculate the probability distributions $P(C)$ versus $C / \bar{C}$ for a given $P_{\infty}$ where $\bar{C}$ is the average value of $C$ and the results are plotted in Fig. 3. In this case, $C^{*}$, the most probable value of $C$, is determined by the fixed infinite cluster size $\mathrm{P}_{\infty}$ with $\mathrm{C}^{*} \approx \mathrm{P}_{\infty}^{2}$, and the broadness of $\mathrm{p}(\mathrm{C})$ comes from the presence of clusters other than the infinite one. Because the largest cluster size is fixed, the upper cutoff of $p(C)$ emerges due to the limitation on the sizes of other clusters that by definition must be smaller than the largest cluster. For the RR strategy, the broadness of $p(C)$ for ER network is bigger than that of SF networks at the same $P_{\infty}$, especially for $\lambda=2.5$ where the system is always high above criticality and the variation is relatively small. On the contrary, for the HDR strategy, the broadness of $p(C)$ for ER and SF networks are of the same order due to the fact that for HDR, $\mathrm{p}_{\mathrm{c}}$ is also finite for $\lambda=2.5$. This observation is consistent with the results shown in Fig. 2.

The broadness of $\mathrm{p}(\mathrm{C})$ for fixed $\mathrm{P}_{\infty}$ is quantitatively characterized by its standard deviation $\sigma_{\mathrm{C}}$. Fig. 4(a) shows the relative standard deviation $\sigma_{\mathrm{C}} / \bar{C}$ for the RR strategy in ER networks, where $\bar{C}$ is the average value of $C$. For increasing value of $P_{\infty}$, the infinite cluster gradually gains control of the system and therefore $\sigma_{C} / \bar{C}$ 
a

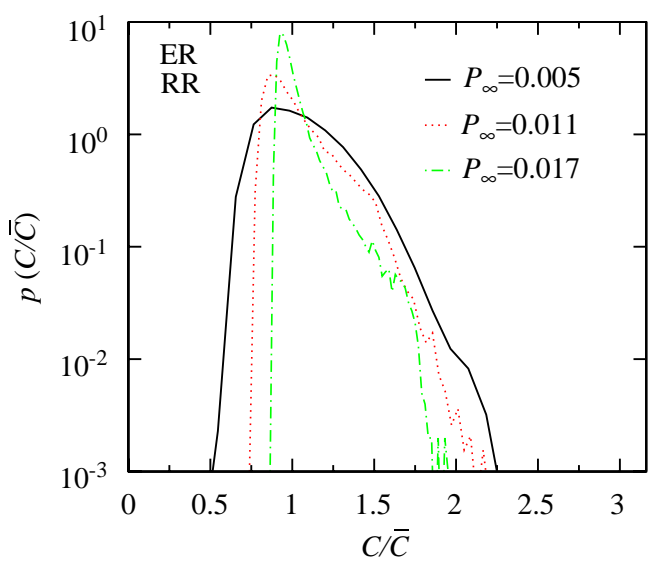

C

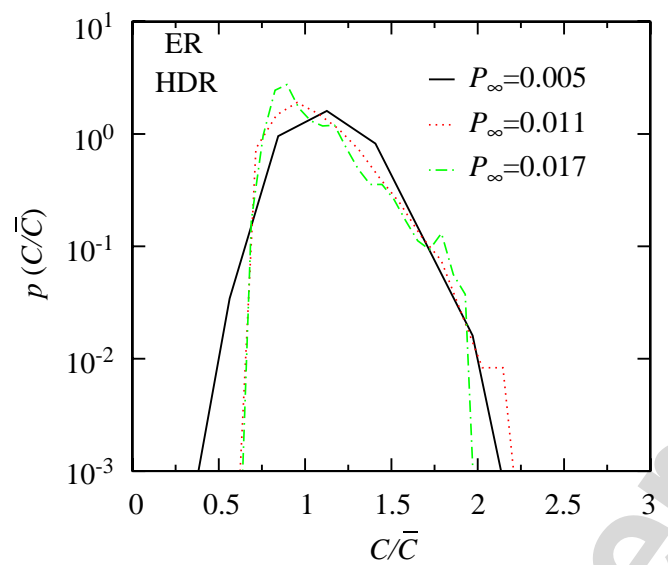

b

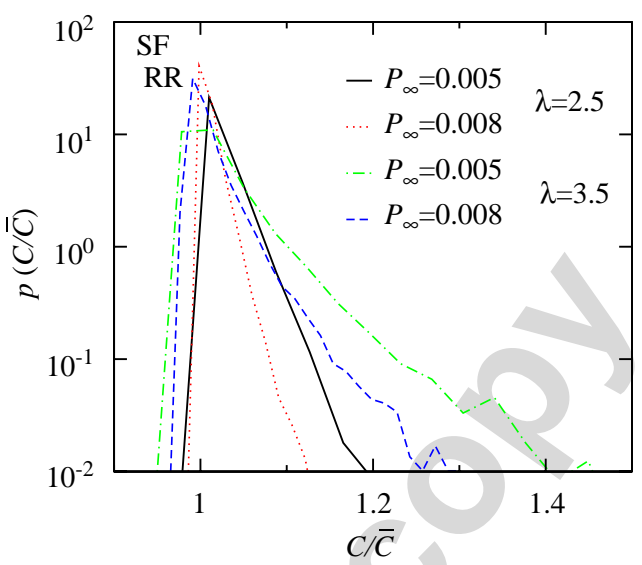

d

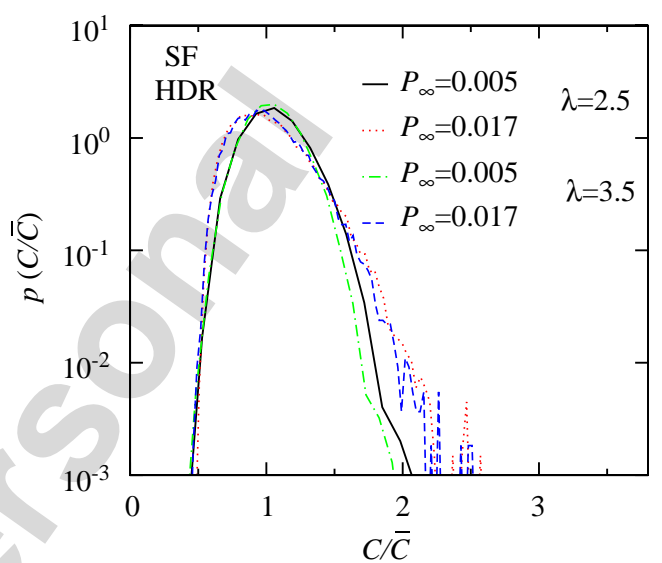

Fig. 3. Probability distributions $p(C / \bar{C})$ versus $C / \bar{C}$ for several values of $P_{\infty}$ and for ER networks with $\langle k\rangle=3, N=200000$ and $S F$ networks with $N=80000$ and $\lambda=2.5$ and 3.5. (a) RR strategy on ER networks, (b) RR strategy on SF networks, (c) HDR strategy on ER networks and (d) HDR strategy on SF networks.
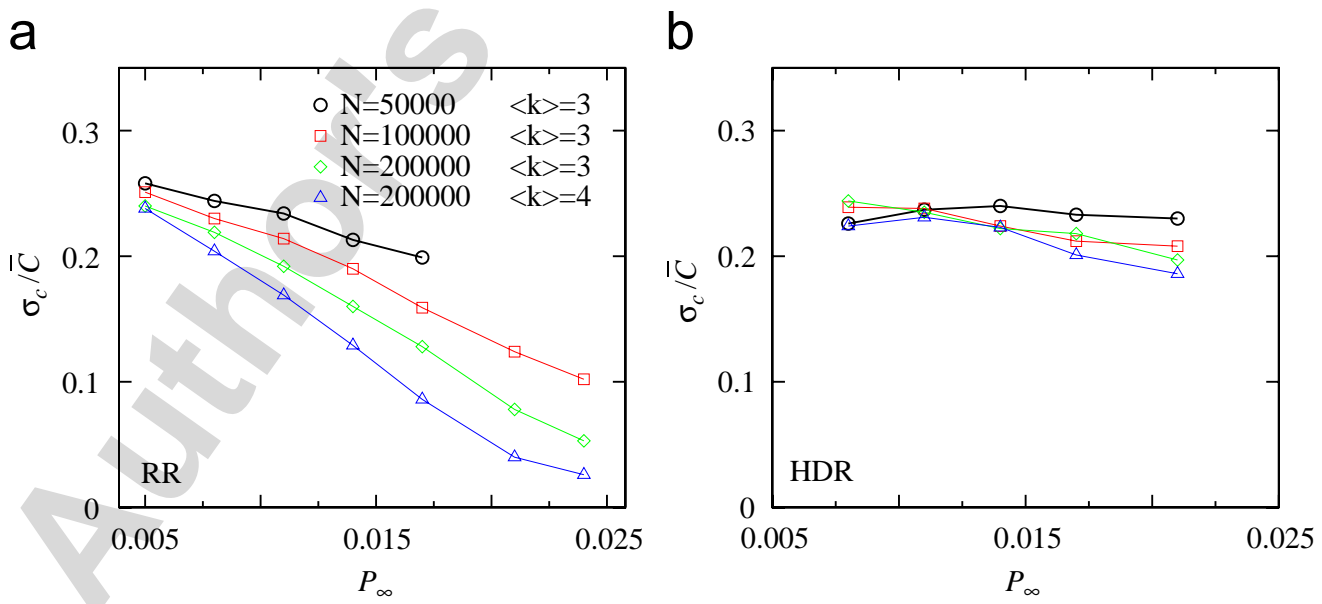

Fig. 4. The dependence of $\sigma_{\mathrm{C}} / \overline{\mathrm{C}}$ for (a) RR and (b) HDR on system size $\mathrm{N}$ and average degree $\langle\mathrm{k}\rangle$ of ER networks.

becomes smaller. It can also be observed that $\sigma_{\mathrm{C}}$ is smaller for larger system sizes $\mathrm{N}$ and larger $\langle\mathrm{k}\rangle$. The result for the HDR strategy is shown in Fig. 4(b) and one can observe that in this case, the relative standard deviation of $C$ is much less sensitive to the value of $P_{\infty}$, as expected in Fig. 3. 
a

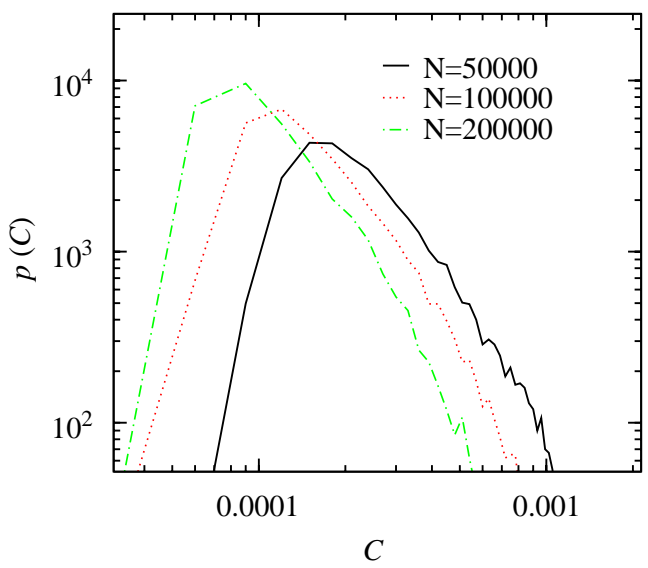

b

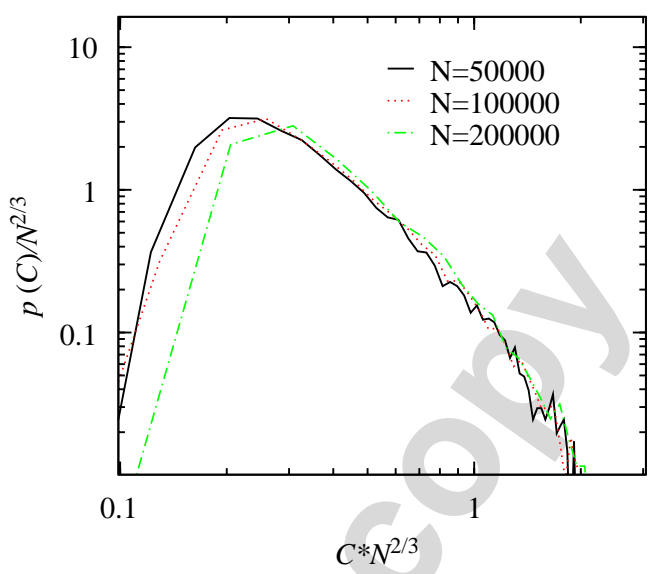

Fig. 5. The dependence of $p(C)$ on the system size $N$ with $p=p_{c}$ for (a) before scaling and (b) after scaling. Simulations are performed on ER networks with $\langle k\rangle=3$.

Now we focus on the dependence of $p(C)$ on the system size $N$ at $p_{c}$ (Fig. 5). From percolation theory and for ER under RR strategy, the infinite cluster size $N_{\infty}$ at criticality behaves as $[23,24]$

$$
\mathrm{N}_{\infty} \sim \mathrm{N}^{2 / 3} \text {. }
$$

Since $C$ follows similar behavior as $N_{\infty}$ at criticality, we expect $C$ for $p=p_{c}$ to behave as,

$$
\mathrm{C} \equiv 1-\mathrm{F} \approx\left(\mathrm{N}_{\infty} / \mathrm{N}\right)^{2} \sim \mathrm{N}^{-2 / 3} \text {. }
$$

Thus, we expect the probability distribution $p(C)$ with $p=p_{c}$ to scale as

$$
\mathrm{p}(\mathrm{C})=\mathrm{N}^{2 / 3} \mathrm{~g}\left(\mathrm{CN}^{2 / 3}\right)
$$

where $\mathrm{g}$ is a scaling function.

Fig. 5 supports this scaling relationship. We calculate $p(C)$ for RR strategy at criticality on ER networks with $N$ values of 50000, 100000,200000 and $\langle k\rangle=3$ (shown in Fig. 5a), and find a good collapse when plotted (Fig. 5b) using the scaling form of Eq. (5).

\section{Real networks}

The structure ER networks and SF networks that we have been studying so far are random and only determined by the degree distribution of the network. Research has shown that real networks often exhibit structural properties of importance for the percolation threshold such as high level of clustering, assortativity and fractality that these types of networks do not exhibit $[12,25]$. We therefore test our results about the correlation between $\mathrm{C}$ and $\mathrm{P}_{\infty}$ on a large real social network. The network we use is extracted from a data set obtained from Statistics Sweden [26] and consists of all geographical workplaces in Sweden that can be linked with each other by having at least one employee from each workplace sharing the same household. Household is defined as a married couple or a couple having kids together that are living in the same flat or house. Unmarried couple without kids and other individuals sharing household are not registered in the data set as household. This kind of network has been shown to be of importance for the spreading of influenza [27] and is also likely to be of importance for the spread of information and rumors in society. The network consists of 310136 nodes (workplaces) and 906260 links (employees sharing the same households) and, as shown in Fig. 6(a), is approximately a SF network with $\lambda \approx 2.6$ and an exponential cut off. The network shows almost no degree-correlation (assortativity) preference (Fig. 6(b)). However, the workplace network clustering coefficient $\mathrm{C}$ is significantly higher than the random SF network with same $\lambda$ and $\mathrm{N}$ (Fig. 6(c)). The average of $\mathrm{C}$ is 0.048 for the workplace network versus $3.2 \times 10^{-4}$ for the random SF networks, which is consistent with the 
a

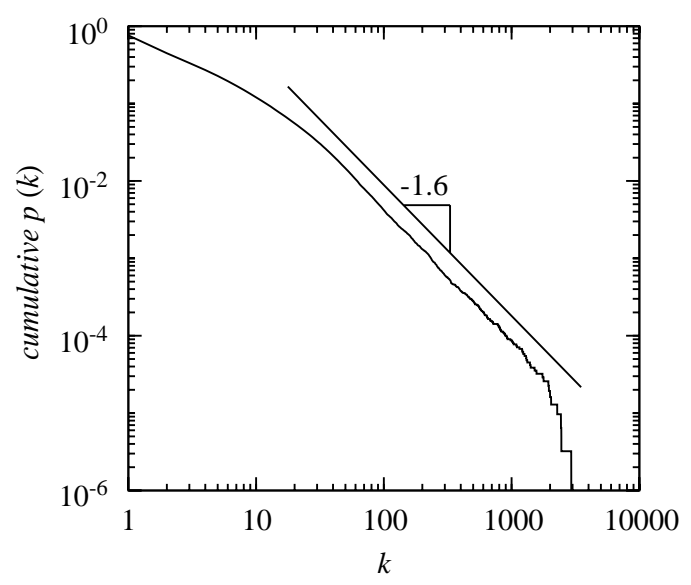

b

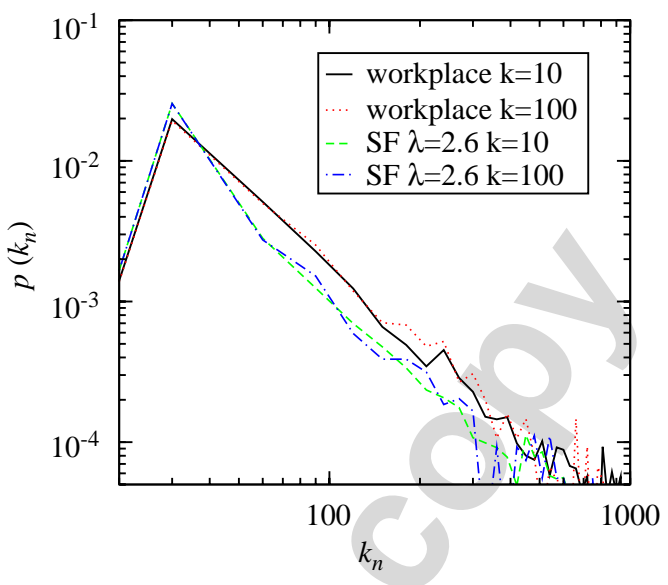

C

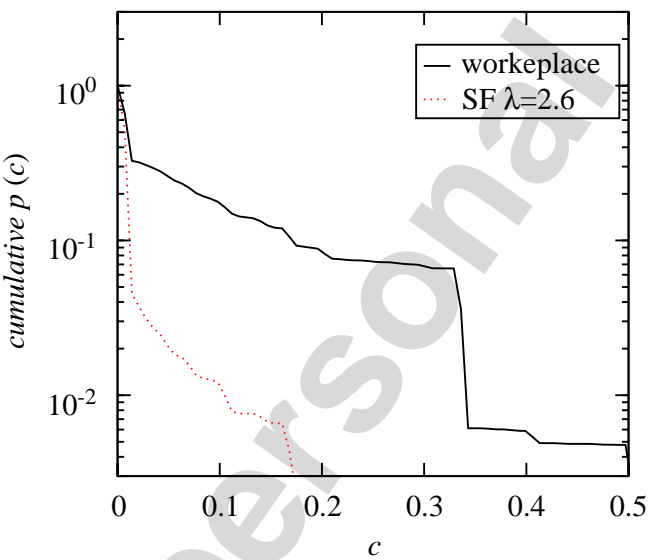

Fig. 6. Properties of the Swedish network of workplaces. (a) The cumulative degree distribution (showing $\lambda=2.6$ ). (b) The distribution of $k_{n}$, the degree of the neighbors of nodes having degree k. (c) The cumulative distribution of clustering coefficient c. In (b) and (c) the distributions of random SF networks with the same $\lambda$ and $\mathrm{N}$ are plotted for comparison.

earlier social network studies [28,29]. Fig. 7(a) and (b) shows simulation results for several values of $p$ for $P_{\infty}$ versus $C^{1 / 2}$. The curves are linear, similar to Fig. 2 for our model networks. Moreover, Fig. 7(c) and (d) shows that $C^{1 / 2}$ and $P_{\infty}$ are almost identical above the criticality threshold $p_{c}$ for a typical configuration after either RR and HDR. For $p$ below criticality, differences appear which are especially obvious for HDR strategy where $q_{c}=1-p_{c}$ is relatively small. While $P_{\infty}$ rapidly decreases to a very small value (below $10^{-5}$ ), a plateau shows up in the curve of $C^{1 / 2}$ due to the influence of the small clusters.

\section{Summary}

In summary, we study the measure for fragmentation $F \equiv 1-C$ proposed in social sciences and relate it to the traditional $\mathrm{P}_{\infty}$ used in percolation theory. For $p$ above criticality, $C$ and $\mathrm{P}_{\infty}$ are highly correlated and $C \approx P_{\infty}^{2}$. Close to criticality, for $p \geqslant p_{c}$ and below $p_{c}$, variations between $C$ and $P_{\infty}$ emerge due to the presence of the small clusters. For systems close to or below criticality, $F$ gives better precision for fragmentation of the whole system compared to $P_{\infty}$. We study the probability distribution $p(C)$ for a given $P_{\infty}$ and find that $p(C)$ at $p=p_{c}$ obeys the scaling relationship $p(C)=N^{2 / 3} g\left(C N^{2 / 3}\right)$ for both $R R$ strategy on ER network, and for HDR on scale free networks. For an alternative measure of connectivity of networks see Ref. [30]. 
a

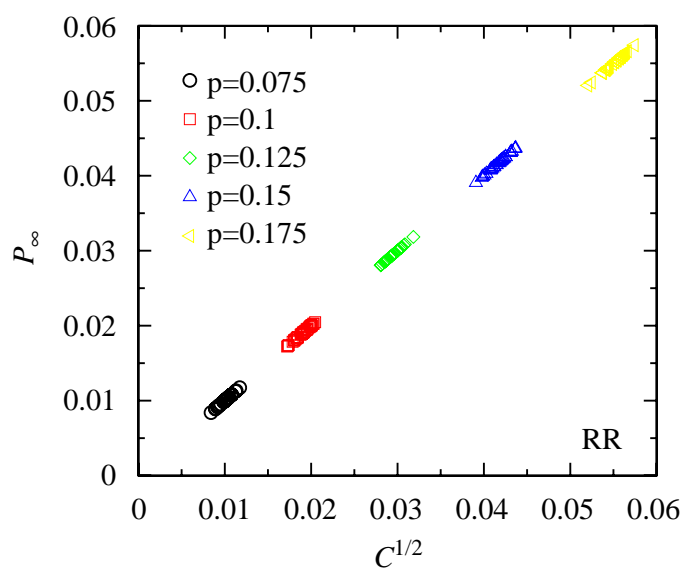

C

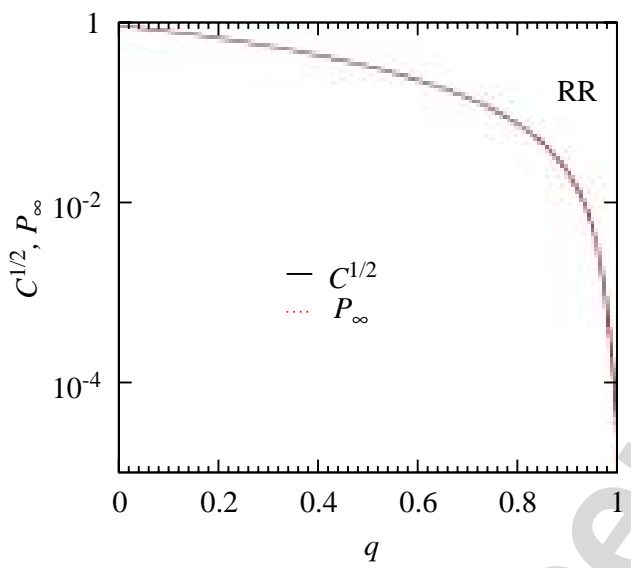

b

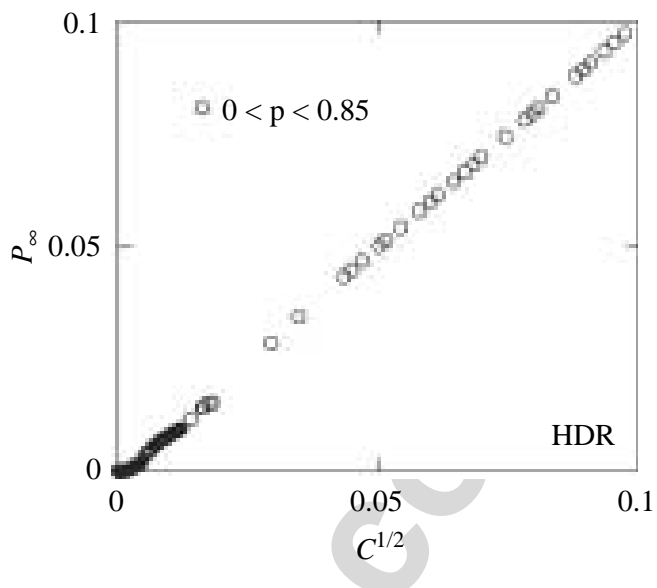

d

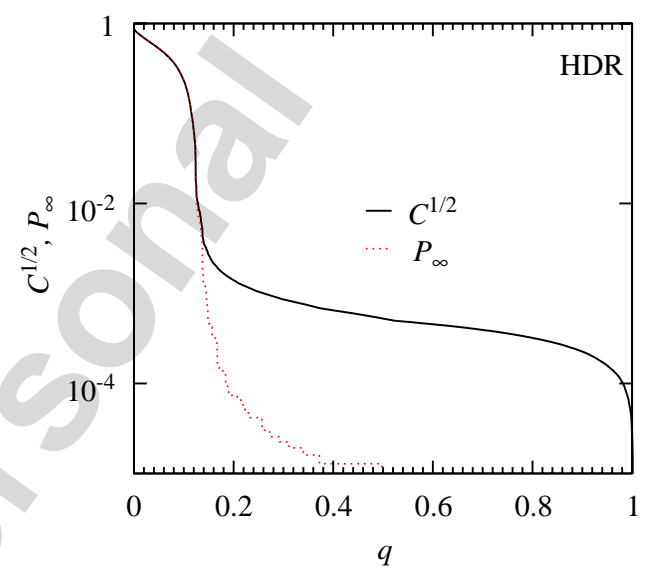

Fig. 7. $P_{\infty}$ versus $C^{1 / 2}$ for (a) RR strategy and (b) HDR strategy and plot $C^{1 / 2}, P_{\infty}$ versus $q$ for (c) RR strategy and (d) HDR strategy for the Swedish network of workplaces with $\mathrm{N}=310136$ nodes.

\section{Acknowledgments}

We thank ONR, European NEST project DYSONET, and Israel Science Foundation for financial support.

\section{References}

[1] G. Paul, S. Sreenivasan, H.E. Stanley, Phys. Rev. E 72 (2005) 056130.

[2] R. Albert, H. Jeong, A.-L. Barabási, Nature, London 406 (2000) 378.

[3] M.E.J. Newman, Phys. Rev. Lett. 89 (2002) 208701.

[4] V. Paxon, IEEE/ACM Trans. Networking 5 (1997) 601.

[5] R. Cohen, K. Erez, D. Ben-Avraham, S. Havlin, Phys. Rev. Lett. 85 (2000) 4626.

[6] D.S. Callaway, M.E.J. Newmann, S.H. Strogatz, D.J. Watts, Phys. Rev. Lett. 85 (2000) 5468.

[7] R. Cohen, et al., Phys. Rev. Lett. 86 (2001) 3682.

[8] A. Valente, A. Sarkar, H.A. Stone, Phys. Rev. Lett. 92 (2004) 118702.

[9] G. Paul, T. Tanizawa, S. Havlin, H.E. Stanley, Eur. Phys. J. B 38 (2004) 187.

[10] F. Chung, L. Lu, Ann. Combinatorics 6 (2002) 125.

[11] Z. Burda, A. Krzywicki, Phys. Rev. E 67 (2003) 046118.

[12] C. Song, et al., Nature 433 (2005) 392.

[13] L.C. Freeman, The Development of Social Network Analysis: A Study in the Sociology of Science, Empirical, 2004.

[14] S. Wasserman, K. Faust, D. Iacobucci, M. Granovetter, Social Network Analysis: Methods and Applications, Cambridge, 1994.

[15] T. Tanizawa, G. Paul, R. Cohen, S. Havlin, H.E. Stanley, Phys. Rev. E 71 (2005) 047101.

[16] R. Pastor-Satorras, A. Vespignani, Phys. Rev. E 65 (2002) 036104. 
[17] P. Holme, B.J. Kim, C.N. Yoon, S.K. Han, Phys. Rev. E 65 (2002) 056109.

[18] S.P. Borgatti, Comp. Math. Org. Theory 12 (2006) 21.

[19] Group of connected nodes known as "component" in the language of sociology.

[20] A. Bunde, S. Havlin, Fractals and Disordered Systems, Springer, Berlin, 1995.

[21] D. Stauffer, A. Aharony, Introduction to Percolation Theory, Taylor \& Francis, London, 1994.

[22] R. Cohen, et al., Phys. Rev. E 66 (2002) 036113.

[23] P. Erdős, A. Rényi, Publ. Math. (Debrecen) 6 (1959) 290.

[24] R. Cohen, S. Havlin, D. Ben-Avraham, Structural properties of scale free networks, in: S. Bornholdt, H.G. Schuster (Eds.), Handbook of Graphs and Networks, vol. 4, Wiley-VCH, New York, 2002.

[25] M.E.J. Newman, SIAM Rev. 45 (2003) 167.

[26] 〈WWW.SCB.SE〉.

[27] C. Viboud, O.N. Bjørnstad, D.L. Smith, L. Simonsen, M.A. Miller, B.T. Grenfell, Science 312 (2006) 447.

[28] G. Csányi, B. Szendrői, Phys. Rev. E 69 (2004) 036131.

[29] K. Klemm, V.M. Eguíluz, Phys. Rev. E 65 (2002) 057102.

[30] E.J. Bienenstock, Balancing Efficiency and Vulnerability in Social Networks, this volume. 